\title{
Electroacupuncture to treat with Overactive Bladder in Postmenopausal Women: study protocol for a multicenter, randomized, controlled, parallel clinical trial
}

\author{
Eun-Young Nam¹', Ju-Yeon Park², Junyoung Jo ${ }^{2,3}$, Seung-Hyun Jung ${ }^{4}$, Chi-Yeon Lim ${ }^{5}$ and Dong-II Kim ${ }^{6 *}$
}

\begin{abstract}
Background: Electroacupuncture has been used for treatment in patients with overactive bladder. This study was conducted to evaluate the efficacy and safety of electroacupuncture for treating overactive bladder of postmenopausal women.

Methods/design: This is a multicenter, randomized controlled, parallel clinical trial. Two hundred ninety participants with overactive bladder syndrome will be recruited from Dongguk University Bundang Oriental Hospital and Cheonan Korean Medicine Hospital of Daejeon University and randomly allocated into one of two groups in a 1:1 ratio. One group will receive electroacupuncture (EA) and the other acupuncture (AC). The allocation will be concealed from both participants and assessors. The study period will be about 10 weeks, including 6 weeks of electroacupuncture or acupuncture treatment and a four week follow-up period. Both EA group and AT group will undergo acupuncture at 7 fixed points, and the EA group will undergo electronic stimulation at 6 points. The primary outcome will be the average number of micturitions per $24 \mathrm{~h}$ based on a 3-day bladder diary. The secondary outcome will comprise the 3day bladder diary, the overactive bladder symptom score and the results of the King's health questionnaire.
\end{abstract}

Discussion: The results of this trial will provide information regarding the efficacy and safety of electroacupuncture for treating overactive bladder in postmenopausal women.

Trial registration: ClinicalTrials.gov, NCT03260907. Registered on 24 August 2017.

Keywords: Overactive bladder, Menopause, Postmenopausal overactive bladder, Electroacupuncture

\section{Background}

Overactive bladder $(\mathrm{OAB})$ is a highly prevalent disorder that has a negative impact on quality of life $[1,2]$. OAB is defined as urinary urgency, usually accompanied by frequency and nocturia, with or without urgent urinary incontinence, in the absence of urinary tract infection (UTI) or other obvious pathology [3-5]. In patients with $\mathrm{OAB}$, frequency $(85 \%)$ is the most commonly reported symptom, followed by urgency $(54 \%)$ and urge incontinence (36\%) [6].

\footnotetext{
* Correspondence: obgykdi@hanmail.net; obgykdi@daum.net

${ }^{6}$ Department of Obstetrics \& Gynecology, College of Korean Medicine,

Dongguk University Korean Medicine Hospital, 27 Dongguk-ro, Ilsandong-gu, Goyang-si, Gyeonggi-do 10326, Republic of Korea

Full list of author information is available at the end of the article
}

The condition is more common in women and in people over 40 years of age $[2,3]$. The prevalence of $\mathrm{OAB}$ increases with age and duration of menopause [6]. The overall prevalence of $\mathrm{OAB}$ in Korean women has been shown to be $14.3 \%$, while it was $18.4 \%$ for those aged over $40[7,8]$.

Acupuncture, which is one of the primary treatments utilized traditional Korean medicine (TKM), involves various stimulation methods such as electrical devices, moxibustion, pharmacopuncture, and thread-embedding therapy to stimulate acupoints, for controlling bladder function at the lower abdomen and lumbar-sacral area [8]. Among these methods, electroacupuncture is a typical and common treatment for $\mathrm{OAB}$ in TKM, which is effective at suppressing excessive 
contractions and activities of the detrusor muscle, improving bladder compliance, maintaining normal urination, and improving pathological changes in bladder tissue $[9,10]$. Mechanical stimulation is supposed to send signals to the spinal cord via the sensory ganglia and interneurons, thereby regulating the activity of motor neurons in the brain stem that control autonomic functions, including urinary activity [11, 12]. However, the efficacy and safety of electroacupuncture are still controversial.

Recent clinical studies have evaluated the efficacy electric stimulation for treating $\mathrm{OAB}$ [13] However, a randomized controlled trial (RCT) for validation of the comparative efficacy and safety of acupuncture with electric stimulation as concurrent treatment has not yet been undertaken.

This study will evaluate the efficacy and safety of electroacupuncture for treating $\mathrm{OAB}$ of menopausal women.

\section{Methods/design}

\section{Study design}

This is a multicenter, randomized controlled, parallel clinical trial. The study protocol conforms to the Consolidated Standards of Reporting Trials (CONSORT) [14] and Standards for Reporting Interventions in Clinical Trials of Acupuncture (STRICTA) [15] guidelines. The study process is illustrated in Fig. 1. The institutional review board of Dongguk University Bundang Oriental Medical Hospital approved the study (2017-0008). The protocol has been registered in ClinicalTrial.gov (NCT03260907).

Participants will be recruited through advertisements in outdoor areas as well as on hospital websites. Two hundred ninety participants with overactive bladder syndrome will be randomly allocated to one of two in a 1:1 ratio. One group will be the electroacupuncture (EA) group, and the other will be the acupuncture (AC) group. The

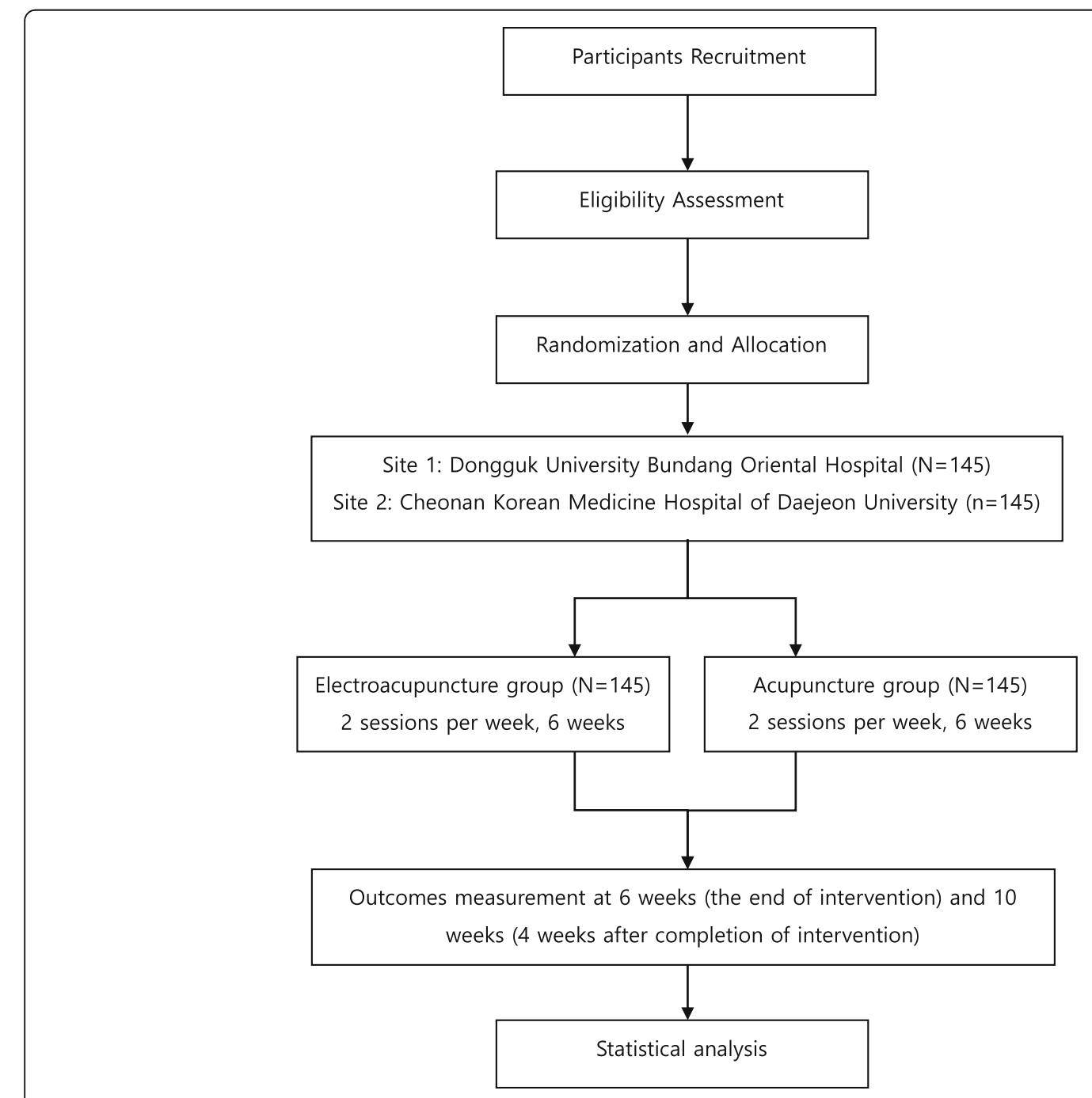

Fig. 1 Flowchart of the study process 
study period will be about 10 weeks, including 6 weeks of electroacupuncture or acupuncture treatment and a 4 week follow-up period. The schedules for enrollment, intervention, and assessments is shown in Table 1. The SPIRIT checklist is provided in Additional file 1.

\section{Eligibility criteria}

Two hundred ninety patients with $\mathrm{OAB}$ will be recruited from two hospitals in Korea: Dongguk University Bundang Oriental Hospital in Bundang, and Cheonan Korean Medicine Hospital of Daejeon University in Cheonan. After obtaining informed consent from the patients or their representatives, we will screen them according to the eligibility criteria for enrollment in the study.

\section{Inclusion criteria}

The inclusion criteria for the study will be as follows: (1) women over 40 years of age without the possibility of pregnancy; (2) those who have a history of amenorrhea for at least 1 year and have no previous history of hormone replacement therapy for the last 6 months; (3) those who have symptoms of urinary frequency and urgency lasting more than 3 months; (4) those who fit the diagnostic criteria for $\mathrm{OAB}$, with a total score of more than 3 points on the Korean version of the Overactive Bladder Symptom Score (OABSS); (5) those who have an average urinary frequency of more than eight times per day and urgency defined by the urgency rating scale (URS) on the bladder diary of more than 2 points and/or urgent urinary incontinence (UUI) on the 3-day bladder diary during a 1-week screening period; (6) those who agree to this clinical study after sufficient explanation.

\section{Exclusion criteria}

The exclusion criteria for the study are as follows: (1) diagnosed with UTI by urine examination; (2) having stress urinary incontinence without symptoms of OAB; (3) suspected of having voiding dysfunction induced by neurological damage; (4) a medical history of cystocele, uterine prolapse or similar condition; (5) a medical history of obstructive uropathy such as urinary stones and urinary tumors; (6) a surgical history of urethra or bladder; (7) a medical history of malignant tumors of the urinary tract; (8) a medical history of neurological disease or psychiatric illness; (9) an artificial cardiac pacemaker or implantable cardioverter defibrillator in the chest; (10) having experienced a hypersensitivity reaction after an acupuncture treatment, or showing any other contraindications; (11) having participated in another clinical trial within the past three months; (12) having taken therapeutic drugs that may affect bladder function within one month of the start of this study; (13) having inadequate literacy to complete the study documents.

Table 1 Schedule to be used for enrolment, intervention, and assessments. OABSS, overactive bladder symptom score; KHQ, King's health questionnaire

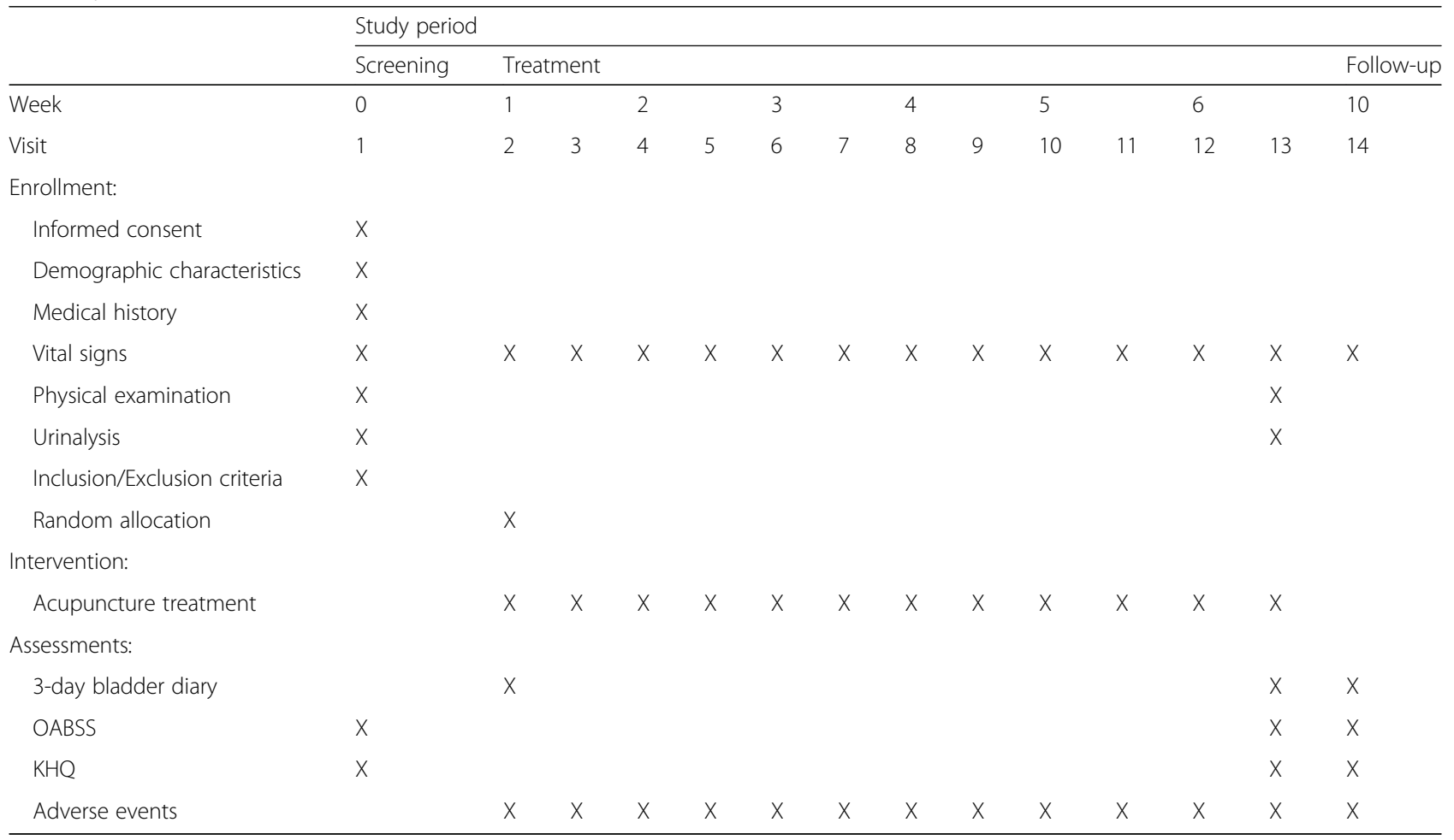




\section{Dropout criteria}

Patients will be removed from the study if they are unwilling to continue their participation in the study. Patients who fail or anticipate that they might fail to attend at least 10 sessions will also be dropped from the study.

\section{Randomization, allocation concealment and blinding}

A table of random numbers will be produced according to sample size using the statistical software SAS (version 9.4; SAS Institute Inc., Cary, NC, USA), and sealed in opaque envelopes. The envelopes will be delivered to each research center and stored in a double-locked cabinet. The practitioner will open the corresponding envelope and allocate the participants who meet the eligibility criteria after signing an informed consent form. Participants will be randomly allocated to the electroacupuncture (EA) group or the acupuncture (AC) group at a ratio of 1:1. After the practitioner checks the group allocation for the subject, the envelope will be stored again in a separate, double-locked cabinet.

Randomization will be stratified by centers and is conducted with concealment of the randomization list.

Practitioners cannot be blinded, and they will be excluded from the assessment procedure. Outcomes will be assessed by researchers who are not involved in the intervention procedure.

\section{Interventions}

The intervention treatment period includes 12 sessions over 6 weeks. The participants will visit the research center twice a week through the 6 week period. Licensed Korean medical doctors who have at least 1 year of clinical experience will perform the intervention. Detailed information regarding the schedule of the enrolment, interventions, and assessments is provided in Additional file 1 and Table 1.

For the EA and AC groups, disposable, sterilized, filiform needles (diameter $0.25 \mathrm{~mm}$, length $40 \mathrm{~mm}$; Dong-Bang Acupuncture Inc., Seoul, Korea) will be inserted into the skin at the following acupoints: unilaterally at CV3, CV4, and GV20, bilaterally at KI3 and SP6. When inserting at CV3, the needling direction will be toward CV2. The needles will be inserted to a depth of between 5 and $30 \mathrm{~mm}$, and the acupuncture needles will be manipulated to achieve de qi and retained for $20 \mathrm{~min}$.

In the EA group, electrical stimulation will be performed simultaneously during the retaining period. An electric acupuncture device (CellMac STN-110, Stratek Co., Republic of Korea) will be connected to the CV3-CV4 and KI3-SP6 acupoints and deliver stimulation with a $2 \mathrm{~Hz}$ frequency, asymmetric bimodal pulse, continuous wave mode, and a maximum intensity below the threshold (7.6-13.9 mA). Electrical stimulation will be delivered at an intensity that the participant can notice but will still feel comfortable with.

\section{Prohibited and permitted concomitant treatment}

Participants will be prohibited from receiving any $\mathrm{OAB}$ treatment outside of the interventions of this trial during the 6-week treatment period. If a patient start medication for $\mathrm{OAB}$ during the treatment period, they will be withdrawn from the trial. All new treatments started after the beginning of the trial and concomitant medications to treat medical conditions unrelated to OAB will be recorded on the Case Report Form (CRF).

\section{Outcomes}

Although it is impossible to ensure blinding for intervention conductor and the participants, we will ensure blinding for the outcome measurements analyses by independent assessors unaware of group allocation. The following outcomes will be assessed by independent assessors blinded to the allocation. Efficacy will be assessed by the 3-day bladder diary, the OABSS and the KHQ.

\section{Primary outcome}

The primary outcome measurement of this study is the average number of micturitions per $24 \mathrm{~h}$ based on the 3 -day bladder diary at the end of the treatment period (week 6).

Participants will complete a 3-day bladder diary before and after treatment (week 6), and the follow-up period (week 10). Entries will be recorded in micturition diaries for 3 days before each follow up visit. Participants will record the time of every micturition and rate the intensity of urgency using the five-point URS ( $1=$ no urgency, $2=$ mild urgency, $3=$ moderate urgency, $4=$ severe urgency, $5=\mathrm{UUI})$.

\section{Secondary outcome}

Secondary outcome measurements will include the number of micturitions in the 3-day bladder diary at the end of the treatment period (week 6) and the follow-up period (week 10). The same version of the 3-day bladder diary will be applied again. This includes daytime micturitions per $24 \mathrm{~h}$, nocturnal micturitions per $24 \mathrm{~h}$, total count of urgency (sum of urgency episodes defined as URS $\geq 3$ for three days), total urgency score (sum of urgency score for three days), and total count of UUI (sum of UUI episodes for three days).

In addition, the OABSS and the KHQ will be measured as secondary outcomes. Participants will complete the Korean version of the OABSS and the KHQ at baseline, the end of the treatment period (week 6), and the follow-up period (week 10).

The OABSS is an assessment tool for OAB symptoms that was developed and validated in Japanese 
populations in 2006 [16]. The OABSS consists of four questions regarding $\mathrm{OAB}$ symptoms; daytime frequency, nocturia, urgency, and UUI. The sum of the four scores runs between 0 and 15. The diagnostic criteria for $O A B$ is a total OABSS of $\geq 3$ with an urgency score for question 3 of $\geq 2$ [17].

The KHQ is an assessment tool for quality of life data of OAB symptoms that was developed by Kelleher and colleagues $[18,19]$. The KHQ is a urine questionnaire that can assess the severity of urination symptoms and evaluate the impact of urination symptoms on quality of life. This questionnaire is known to have reliability and validity for measuring the quality of life of patients with incontinence [20]. The 10 domains from the KHQ evaluated are general health perception, incontinence impact, role limitations, physical limitations, social limitations, personal relationships, emotions, sleep/energy, severity measures, and symptom severity.

\section{Safety assessment}

All participants will be monitored for adverse events during the study. Adverse events (AEs) indicate undesirable and unintentional signs, symptoms, or diseases that develop after intervention during the period of a clinical trial. They do not necessarily have a causal relationship with the relevant intervention. Investigators will check participants' vital signs and examine for manifestation of AEs at each visit. Investigators will also conduct physical examinations and laboratory tests, including evaluation of urinalysis (specific gravity, nitrite, $\mathrm{pH}$, protein, glucose, ketones, urobilinogen, bilirubin, blood, white blood cells, urine HCG) at the screening as well as at the end of treatment period (week 6). The outcome assessors will judge the severity (none, mild, moderate, or severe), seriousness, and the correlation between AEs and intervention (definitely related, appears to be related, possibly related, appears to be unrelated, definitely not related, or unclear).

\section{Sample size}

The primary outcome of this study will be the average number of micturitions per $24 \mathrm{~h}$ after the treatment. Based on a previous study [21], we estimated that the mean difference in the pre- and post-treatment change in the average number of micturitions per $24 \mathrm{~h}$ scores between the EA and AC group will be 0.8 and the standard deviation will be 2.1 . With a $5 \%$ significance level and $80 \%$ power, the needed sample size was calculated to be 116 per group. Considering a $20 \%$ drop-out rate, 145 participants are required per group. In conclusion, a total of 290 participants are needed in this trial.

\section{Statistical analysis}

We will analyze results primarily based on the principle of intention-to-treat (ITT) and subordinately based on the per-protocol (PP) principle. The ITT analysis will include any participants who are randomly allocated and have at least one EA or AC session. The PP analysis will cover only patients who have completed at least 10 sessions of EA or AC treatments with no use of medication or medical device of UTI or OAB, and have no serious violation. The primary and secondary continuous outcomes will be compared between randomized groups using ANCOVA models that includes baseline measurement as covariates.

The categorical variables will be analyzed by the Pearson $x^{2}$ test or Fisher's exact test. If there is a significant difference between groups in terms of baseline characteristics, analysis of covariance (ANCOVA) or logistic regression for further evaluation will be performed. When it is necessary to control important variables that may affect the final evaluation, stratification analysis (Cochran-Mantel-Haenszel method, etc.) will be applied.

\section{Discussion}

This will be a multicenter, randomized controlled, parallel clinical study designed to evaluate the efficacy and safety of electroacupuncture treatment of postmenopausal women with $\mathrm{OAB}$, as well as to clarify the effects of electro stimulation of acupuncture on urinary activity. According to recent studies, insufficient evidence exists regarding the efficacy of EA in patients with OAB. Electro-stimulation (ES) is likely to improve $\mathrm{OAB}$ symptoms or OAB-related quality of life more than no active treatment, but it is uncertain whether ES is better than other treatments, such as pelvic floor muscle training (PFMT) or drug therapy [17]. However, percutaneous posterior tibial nerve stimulation (PTNS), which is a kind of electroacupuncture at SP 6, is generally examined as effective treatment option of $\mathrm{OAB}[22,23]$. Moreover, the studies suggest that electroacupuncture compares favorably to drug therapy with a viable long term effect [24-26].

Overactive bladder is an asymptomatic diagnosis that has been defined as comprising symptoms of frequency of more than eight micturitions per $24 \mathrm{~h}$, urgency and urge incontinence, occurring singly or in combination and not explained by metabolic conditions such as diabetes or local pathological factors such as urinary tract infection (UTI), stones, or interstitial cystitis. Patients with an overactive bladder include those with and without a possible neurological cause for their symptoms [27]. Overactive bladder is a chronic condition defined urodynamically as detrusor overactivity that is characterized by involuntary bladder contractions during the filling phase of the micturition cycle [28]. The involuntary contractions result in reduced functional bladder capacity and unpredictable, troublesome symptoms [7]. In clinics, the diagnosis of $\mathrm{OAB}$ is based on urinary symptoms, detailed history, overactive bladder symptom score 
(OABSS), bladder diary, and physical examination. For clinical examination, urinalysis is performed to rule out UTI, and ultrasonography is considered if a female genital tumor is suspected.

The investigators will target postmenopausal women with OAB. After treatment in two groups (electroacupuncture or acupuncture), the investigators will compare urinary symptoms assessed based on the 3-day bladder diary and overactive bladder symptom score (OABSS) as well as the quality of life assessed by King's Health Questionnaire (KHQ) and evaluate the safety of abnormal reaction during the treatment.

In this study, the EA group and AT group will undergo acupuncture at 7 fixed points, and the EA group will include electronic stimulation at 6 points. The efficacy of electric stimulation of acupuncture will be compared to that of acupuncture without any stimulation.

The 7 acupoints were identified based on the guidelines published by the World Health Organization. In the abdomen region, CV 3 and CV 4 have been reported to possibly have the ability to recover bladder dysfunction in literature pertaining to traditional Korean medicine, and were shown to have clinical effectiveness for overactive bladder in previous studies $[29,30]$. These acupoints are considered to have a possible mechanism in which acupuncture stimulation directly increases the excitability of the pelvic nerve, which consequently innervates the detrusor muscle $[9,10]$.

The period required for electroacupuncture treatment of OAB is controversial. According to RCTs from Korea and China, about 12-24 weeks are needed to recover and stabilize bladder function with $20-30$ or more sessions of regular and long-term electroacupuncture or acupuncture [9], whereas trials lasting 4-12 weeks with 4-24 or more sessions of PTNS were required in a previous systematic review [17]. Based on previous existing studies and actual clinical practices, this study will employ 12 treatment sessions over 6 weeks.

This study is expected to be the first well-designed RCT to determine the effects of adding electro stimulation to acupuncture treatment on $\mathrm{OAB}$. At the end of this project, the findings will provide the clinical evidence of the efficacy and safety of EA as a treatment for $\mathrm{OAB}$ in postmenopausal women.

\section{Dissemination policy}

We will report the final data to the Ministry of Health \& Welfare through the Korea Health Industry Development Institute. We will also publish the results after study completion.

\section{Trial status}

This trial is proposed. Enrollment and trial procedures are expected to be complete by the end of Dec 2019.

\section{Additional file}

Additional file 1: SPIRIT 2013 Checklist: Recommended items to address in a clinical trial protocol and related documents*. (DOC $130 \mathrm{~kb}$ )

\section{Abbreviations}

AC: Acupuncture; AEs: Adverse events; CRF: Case report form;

EA: Electroacupuncture; IRB: Institutional review board; ITT: Intention-to-treat; KHQ: Korean version of King's Health Questionnaire; OAB: Overactive bladder; OABSS: Overactive bladder symptom score; PP: Per Protocol;

PTNS: percutaneous tibial nerve stimulation; RCT: Randomized controlled trial; SPIRIT: Standard Protocol Items: Recommendations for Interventional Trials; STRICTA: Standards for Reporting Interventions in Clinical Trials of Acupuncture; TKM: Traditional Korean medicine; UTI: Urinary tract infection; UUI: Urge urinary incontinence

\section{Funding}

This study was supported by a grant from the Korean Health Technology R\&D Project, Ministry of Health \& Welfare, Republic of Korea. (HB16C0062).

\section{Authors' contributions}

DI Kim conceived of the project, obtained the funding, developed the trial protocol, and is in charge of the whole implementation process. EY Nam contributed to the conception and design of the study, manuscript writing, and critical revision. JY Park and J Jo provided technical advice and made critical revisions. SH Jung is in charge of the recruitment of participants and the arrangement of the whole study process as a principal investigator. $\mathrm{CY}$ Lim was the trial analyst, contributing to the development of statistical analysis plans. All authors read and approved the final manuscript before submission.

\section{Ethics approval and consent to participate}

The study protocol was approved by the Institutional Review Boards (IRBs) of Dongguk University Bundang Oriental Hospital (2017-0008). The study procedures will follow the protocols approved by the IRB, and only patients who provide written consent will be included.

Competing interests

The authors declare that they have no competing interests.

\section{Publisher's Note}

Springer Nature remains neutral with regard to jurisdictional claims in published maps and institutional affiliations.

\section{Author details}

${ }^{1}$ Department of Obstetrics \& Gynecology, College of Korean Medicine, Gachon University Dongincheon Gil Korean Medicine Hospital, 21 Keunumul-ro, Jung-gu, Incheon 22318, Republic of Korea. ${ }^{2}$ Research institute of Korean Medicine, College of Korean Medicine, Dongguk University, 32 Dongguk-ro, Ilsandong-gu, Goyang-si, Gyeonggi-do 10326, Republic of Korea. ${ }^{3}$ Department of Obstetrics and Gynecology Conmaul Hospital of Korean Medicine 110 Seochojungang-ro, Seocho-gu Seoul 06634, Republic of Korea. ${ }^{4}$ Department of Internal Medicine, College of Korean Medicine, Dongguk University Bundang Oriental Hospital, 268 Buljeong-ro, Bundang-gu, Seongnam-si, Gyeonggi-do 13601, Republic of Korea. ${ }^{5}$ Department of Biostatistics, College of Medicine, Dongguk University, 32 Dongguk-ro, Ilsandong-gu, Goyang-si, Gyeonggi-do 10326, Republic of Korea.

${ }^{6}$ Department of Obstetrics \& Gynecology, College of Korean Medicine, Dongguk University Korean Medicine Hospital, 27 Dongguk-ro, Ilsandong-gu, Goyang-si, Gyeonggi-do 10326, Republic of Korea.

Received: 6 November 2017 Accepted: 1 June 2018

Published online: 15 September 2018

\section{References}

1. Temml C, Heidler S, Ponholzer A, Madersbacher S. Prevalence of the overactive bladder syndrome by applying the International Continence Society definition. Eur Urol. 2005;48:622-7. 
2. Coyne KS, Sexton CC, Kopp ZS, Ebel-Bitoun C, Milsom I, Chapple C. The impact of overactive bladder on mental health, work productivity and health-related quality of life in the UK and Sweden: results from EpiLUTS. BJU Int. 2011;108:1459-71.

3. Haylen B, de Ridder D, Freeman R, Swift S, Berghmans B, Lee J, Monga A, Petri E, Rizk D, Sand P. An International Urogynecological Association (IUGA)/International Continence Society (ICS) joint report on the terminology for female pelvic floor dysfunction. J Assoc Chart Physiother Womens Health. 2012;110:33.

4. Gormley EA, Lightner DJ, Burgio KL, Chai TC, Clemens JQ, Culkin DJ, Das AK, Foster HE, Scarpero HM, Tessier CD. Diagnosis and treatment of overactive bladder (non-neurogenic) in adults: AUA/SUFU guideline. J Urol. 2012;188: 2455-63.

5. Abrams P, Cardozo L, Fall M, Griffiths D, Rosier P, Ulmsten U, Van Kerrebroeck $P$, Victor A, Wein A. The standardisation of terminology in lower urinary tract function: report from the standardisation sub-committee of the International Continence Society. Urology. 2003;61:37-49.

6. Sang $\mathrm{JH}$, Park HM. Survey on the prevalence of overactive bladder in healthy Korean postmenopausal women. J Kor Soc Menopause. 2012;18:60-6.

7. Milsom I, Abrams P, Cardozo L, Roberts R, Thüroff J, Wein A. How widespread are the symptoms of an overactive bladder and how are they managed? A population-based prevalence study. BJU Int. 2001:87:760-6.

8. Kim Y-J, Kim M-C, Lee C-H, Kim J-U, Yook T-H. The effect of needleembedding therapy and pharmacopuncture therapy on patients with urinary incontinence. J Acupunct Meridian Stud. 2011:4:220-4.

9. Choi M-S, Kim D-I. A Study on Korean Medical Clinical Management of Female Overactive Bladder. J Korean Obstet Gynecol. 2014;27:83-102.

10. Kim D, Choi C, Ahn I, Ryu I, Choi M, Lee Y, Lee MS. Efficacy and safety of Baweidihuang-wan in women with overactive bladder: a randomized, double blind, placebo controlled trial. Int J Clin Exp Med. 2014;7:2744.

11. Han J-S. Acupuncture analgesia: areas of consensus and controversy. Pain. 2011;152:S41-8.

12. Burnstock G. Acupuncture: a novel hypothesis for the involvement of purinergic signalling. Med Hypotheses. 2009;73:470-2.

13. Stewart F, Berghmans $B, B \varnothing K$, Glazener CM. Electrical stimulation with nonimplanted devices for stress urinary incontinence in women. Cochrane Database Syst Rev 2016;10:CD012390.

14. Schulz KF, Altman DG, Moher D. CONSORT 2010 statement: updated guidelines for reporting parallel group randomised trials. BMC Med. 2010;8:18.

15. MacPherson H, Altman DG, Hammerschlag R, Youping L, Taixiang W, White A, Moher D. Revised standards for reporting interventions in clinical trials of acupuncture (STRICTA): extending the CONSORT statement. J Evid Based Med. 2010;3:140-55.

16. Homma Y, Yoshida M, Seki N, Yokoyama O, Kakizaki H, Gotoh M, Yamanishi T, Yamaguchi O, Takeda M, Nishizawa O. Symptom assessment tool for overactive bladder syndrome-overactive bladder symptom score. Urology. 2006:68:318-23.

17. Yamaguchi $\mathrm{O}$, Nishizawa O, Takeda M, Yokoyama O, Homma Y, Kakizaki H, Obara K, Gotoh M, Igawa Y, Seki N. Clinical guidelines for overactive bladder. Int J Urol. 2009;16:126-42.

18. Kelleher CJ, Cardozo LD, Toozs-Hobson PM. Quality of life and urinary incontinence. Curr Opin Obstet Gynecol. 1995;7:404-8.

19. Kelleher C, Cardozo L, Khullar V, Salvatore S. A new questionnaire to assess the quality of life of urinary incontinent women. BJOG. 1997;104:1374-9.

20. Reese PR, Pleil AM, Okano GJ, Kelleher CJ. Multinational study of reliability and validity of the King's Health Questionnaire in patients with overactive bladder. Qual Life Res. 2003;12:427-42.

21. Carmona MVO, Molleja ÁMG, Ríos IL, Torronteras AR, Tamajón VMC, Obrero IG. Neuroestimulación percutánea del nervio tibial posterior frente a neuroestimulación de B 6 (Sanyinjiao) en incontinencia urinaria de urgencia. Revista Internacional de Acupuntura. 2013;7:124-30.

22. van BALKEN MR, Vandoninck V, Gisolf KW, Vergunst $H$, Kiemeney LA, Debruyne FM, Bemelmans BL. Posterior tibial nerve stimulation as neuromodulative treatment of lower urinary tract dysfunction. J Urol. 2001;166:914-8

23. Klingler H, Pycha A, Schmidbauer J, Marberger M. Use of peripheral neuromodulation of the S3 region for treatment of detrusor overactivity: a urodynamic-based study. Urology. 2000;56:766-71.
24. Peters KM, MacDiarmid SA, Wooldridge LS, Leong FC, Shobeiri SA, Rovner ES, Siegel SW, Tate SB, Jarnagin BK, Rosenblatt PL. Randomized trial of percutaneous tibial nerve stimulation versus extended-release tolterodine: results from the overactive bladder innovative therapy trial. J Urol. 2009;182: 1055-61.

25. MacDiarmid SA, Peters KM, Shobeiri SA, Wooldridge LS, Rovner ES, Leong FC, Siegel SW, Tate SB, Feagins BA. Long-term durability of percutaneous tibial nerve stimulation for the treatment of overactive bladder. J Urol. 2010;183:234-40.

26. Vandoninck V, van Balken MR, Agró EF, Petta F, Caltagirone C, Heesakkers JP, Kiemeney LA, Debruyne FM, Bemelmans BL. Posterior tibial nerve stimulation in the treatment of urge incontinence. Neurourol Urodyn. 2003:22:17-23.

27. Abrams $P$, Cardozo L, Fall M, Griffiths D, Rosier P, Ulmsten U, van Kerrebroeck $\mathrm{P}$, Victor $\mathrm{A}$, Wein A. The standardisation of terminology of lower urinary tract function: report from the Standardisation Sub-committee of the International Continence Society. Am J Obstet Gynecol. 2002;187: $116-26$.

28. Abrams P, Blaivas JG, Stanton SL, Andersen JT. The standardisation of terminology of lower urinary tract function. The International Continence Society Conunittee on Standardisation of Terminology. Scand J Urol Nephrol Suppl. 1988;114:5-19.

29. Philp T, Shah P, Worth P. Acupuncture in the treatment of bladder instability. BJU Int. 1988:61:490-3.

30. Paik SH, Han SR, Kwon OJ, Ahn YM, Lee BC, Ahn SY. Acupuncture for the treatment of urinary incontinence: A review of randomized controlled trials. Exp Ther Med. 2013;6:773-80.

\section{Ready to submit your research? Choose BMC and benefit from:}

- fast, convenient online submission

- thorough peer review by experienced researchers in your field

- rapid publication on acceptance

- support for research data, including large and complex data types

- gold Open Access which fosters wider collaboration and increased citations

- maximum visibility for your research: over $100 \mathrm{M}$ website views per year

At BMC, research is always in progress.

Learn more biomedcentral.com/submissions 